\title{
The Smart Grid Concept in Oil \& Gas Industries by a Field Trial of Data Communication in MV Power Lines
}

Leonardo R. M. Castor, Ricardo Natale, João P. Favero, Jair A. L. Silva and Marcelo E. V. Segatto, Department of Electrical Engineering, Universidade Federal do Espírito Santo, Vitória-ES, Brasil e-mail:lrmcastor@gmail.com, jair.silva@ufes.brand segatto@ele.ufes.br

\begin{abstract}
Nowadays, communication and networking technologies are essential for Oil \& Gas industries in order to guarantee reliability, safety and low costs in the producing process. Information access and commands and set-points transmission from/to automation systems are challenging tasks mainly due to distributed production areas and hostile environments. This paper presents a field trial of broadband power line communication over an overhead medium voltage grid as a data transport employing $200 \mathrm{Mb} / \mathrm{s}$ BPL modems. Throughput, frequency response, physical speed and latency are some of the investigated network parameters. Experimental results obtained after propagation through $1.63 \mathrm{~km}$ of a multipath MV power grid reveal the viability of the BPL technology as an access network for video and automation data transport in onshore Oil \& Gas industries.
\end{abstract}

Index Terms - Smart grid, overhead medium voltage, broadband powerline, Oil \& Gas industries.

\section{INTRODUCTION}

One of the main goals of the producing petroleum Industries is the operational cost (OPEX) reduction. The producing process is required to be performed safely and with low costs. Due to human resource employment at the manual operating and controlling producing processes, the cost is significantly increased and the employees are submitted to dangerousness procedures. In this scenario, the automation system process emerges as a good solution that can reduces cost and simplify several industrial requirements such as replacing manual work and elevating both reliability and efficiency in the production. On the other hand, reliable and low cost data communication is considered essential for guarantee remote access to the automation system.

Well known technologies such as fiber optic and narrowband wireless systems are used as solution for automation data transport, but with elevated costs and implantation time. The broadband powerline (BPL) technology appears as a good alternative for data communication due to the already installed and pervasive power distribution infrastructure, especially in onshore Oil \& Gas industries [1], [2], [3]. However, impairments generated by the high selectivity observed in its channel frequency response, by impulsive noise and by frequency interference due to radio broadcasters and electrical devices connected in the same network, represent some challenges to overcome for 
convenient BPL communications [4], [5].

Distribution networks at medium voltage (MV) levels (13.8 kV for example) comprised by overhead multiconductor transmission lines (MTL) can be used not only for remote metering but also for real time bidirectional high data rate applications in a tree-like topology [6], [7]. Interference and impulsive noise over MV distribution power lines are not so powerful when compared to the LV grid, due to quantity and type of electrical connected devices [6], [7]. For this reason, the use of the MV power line as a channel for power line communication (PLC) has gained a widespread investigation interest in industries and academic communities.

The principle behind the PLC technique has emerged since 1838, when a remote measurement system was proposed to monitoring battery levels of sites far from the telegraph system between London and Liverpool, using the electrical wiring for communications purpose [8]. In the 1922 the first carrier frequency system began to operate over high-voltage lines in the frequency range 15-500 $\mathrm{kHz}$ for telemetry applications [9]. In 1950, the first PLC systems were designed to works over medium- and low-voltage electrical networks for the remote switching of public lights or for tariff changes [8]. In this case, the carrier frequency was between $100 \mathrm{~Hz}$ and $1 \mathrm{kHz}$ and a one-directional communications were established via control signals. Later, others narrowband communication systems were designed for applications such as control and telemetry of electrical equipment's like remote meter reading, dynamic tariff control, load management, load profile recording and allowing a bidirectional communication [8] [9]. Nowadays, the PLC technology has been enhanced, bringing improvements in transmission speed (up to $205 \mathrm{Mbit} / \mathrm{s}$ ), security, and service quality, providing high performance transmission for broadband services such as video streaming, internet, cloud access, etc. To improve data transmission, the physical layer uses optimized techniques for coding, modulation, and error correction, resulting in excellent connectivity between devices and good transmission rates over a frequency range of 1 to $50 \mathrm{MHz}$ [8].

The communications over the electrical power supply networks standards has been also improved along of the years. The first narrowband PLC specification was created by CENELEC (European Committee for Electrotechnical Standardization) to operate in a frequency range from 9 to $140 \mathrm{kHz}$ with bidirectional communications over the LV (low voltage) electrical network. In the North America and Japan, the frequency spectrum used was $500 \mathrm{kHz}$. Late, others standard organization, consortiums and associations such as European Telecommunications Standards Institute - Power Line Telecommunications (ETSI-PLT), Home-Plug Power-Line Alliance, Institute of Electrical and Electronics Engineers (IEEE) BPL study group, POWERNET, Open PLC European Research Alliance (OPERA) and Universal Power-Line Association (UPA), emerged for standardization of the broadband power line communication system.

Nowadays, a BPL equipment can transport broadband service over a power grid in a frequency range of 1 to $50 \mathrm{MHz}$ and bandwidth of $30 \mathrm{MHz}$.

In this paper we report the technical feasibility of using the MV grid as a BPL network for 
automation and video data transport in the conception of a smart grid in onshore Oil \& Gas industries. It is shown that the designed network ensures throughput for remote metering, load control and property security of extraction oil-wells. Localized in a rural area, the BPL cell is composed by a signal processing base station connected to three oil-wells by $1.6 \mathrm{~km}$ of overhead MV lines. Equipped with a master BPL modem, the base station is responsible for control and supervision of their relevant process variables in a tree-like topology. Equipped with three-phase mechanical pumping units and with programmable logic controllers, each oil-well contains a slave BPL modem to provide upstream communications. According to our design strategy, BPL gateways coupled to the grid at each $400 \mathrm{~m}$ guarantees the link budget for both downstream and upstream considering the small number of branches that certainly degrades the system performance. Nevertheless, PLC technology, based on this design strategy, represents an attractive and cost-effective solution for remote metering and also for patrimony video monitoring of the three oil-wells equipped with progressive cavity pump units.

Network topology, bandwidth requirements, frequency response, physical layer transmission rate and latency are systematically analyzed in the overhead MV distribution power grid. For this purpose, six PLC modems, configured as master, repeaters and slaves are used to compose a BPL cell over a $1.6 \mathrm{~km}$ overhead MV line.

This paper is organized as follows. In the Section II, the state of the art of a modem based on the Opera open specification for broadband Internet access is showed. Physical and logical trial topologies are described in Section III. Measurements of bandwidth, physical transmission rates and signal spectrums are analyzed in Section IV. Finally, Section V presents the conclusions and final considerations.

\section{BROADBAND POWERLINE BASED ON OPERA SPECIFICATIONS}

A broadband power line access network consists of a number of terminal users that transmit/receive information in a shared medium to/from a centralized station. In a long reach, with different costumer premise equipments (CPE's) connected to a same head end (HE), time division repeaters (TDR's) can be inserted in the network in order to retransmit the signal and thus increase the coverage.

\section{A. Network Architectures}

Fig. 1 depicts common tree-like topologies founded in BPL access networks, where a central node HE concentrates and command all of the upstream and downstream traffic in the network. All kinds of structures in MV and LV power line grids can be reduced to an HE plus repeaters structures [11]. Normally, the HE assigns resources to all nodes of the BPL cell through the use of a token, according to the quality of service (QoS) requirements of the flows circulating on the cell. When connected to an $\mathrm{HE}$ or to another repeater, the TDR acts as a master node, sharing its allocated channel among their slave nodes according to the traffic flows, the BPL service class and the origin and destination. Therefore, it will be the slave of the HE or of another TDR, and the master of its slaves. 


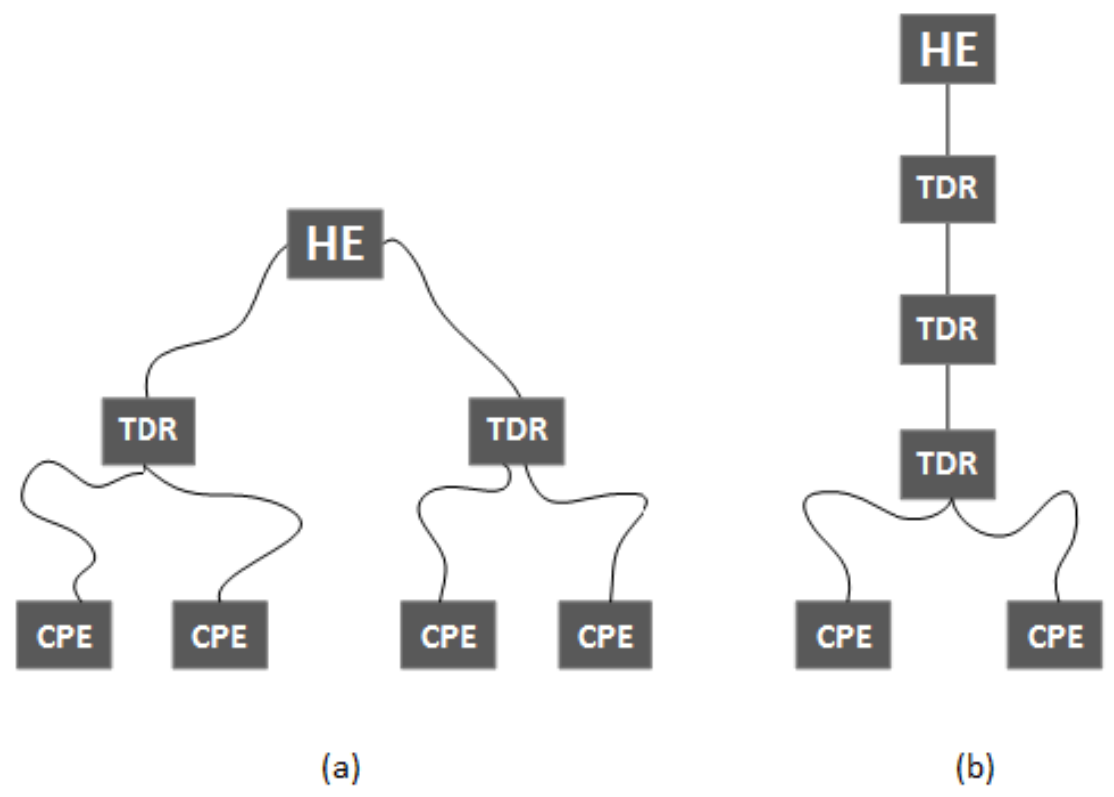

Fig. 1. Common tree-like topologies of BPL access network. HE: Head End, CPE: Customer Premise Equipment, TDR: Time Division Repeater.

CPE's are BPL units installed close to customer's household. As a slave, a CPE must subscribe to the network before being able to access the channel. This means that it must select a master that assigns a channel access time. Then, a validation process occurs in order to acknowledge if the CPE is valid. After being accepted to the network, the CPE automatically downloads a file (autoconfiguration process) detailing its configuration parameters [11].

\section{B. Physical Layer Overview}

One of the most significant characteristics of PLC channels is its frequency selective due to reflections caused by impedance mismatches. The overhead MV power line grid also exhibits attenuation and low-pass behavior which limits the bandwidth that shall be used in the communication [16]. Moreover, the time-varying phenomenon occurs due to changes in the topology, cables and connected loads. To overcome those drawbacks, orthogonal frequency division multiplexing (OFDM) techniques has been implemented in the BPL equipments, with its inherent adaptability in the presence of frequency selective channels, its robustness to impulsive noise and high spectral efficiency. Among its several subcarriers, OFDM can employ different levels of amplitude differential phase shift keying (ADPSK) in an adaptive mapping, after channel estimation through pilot symbols.

The bandwidths of the used BPL modems define three operation modes: $30 \mathrm{MHz}, 20 \mathrm{MHz}$ and 10 MHz. The BPL system used on the field trial operates in $30 \mathrm{MHz}$ signal bandwidth and $10 \mathrm{MHz}$, for data rates up to 205 and $84 \mathrm{Mb} / \mathrm{s}$ respectively.

A BPL node can operate in one of the different bandwidth modes at a given time [11]. The spectrum occupied by signals can change according to the defined signal bandwidth from 2 to 34 MHz. Some of the important parameters of the BPL modems used in the field trial are summarized in Table I. 
TABle I. SOME BPL PARAMETERS SPECIFIEd IN OPERA. PSD: Power SPECTRAL Density.

\begin{tabular}{cccc}
\hline Central Frequency & Bandwidth & PSD & Max. Bit Rate \\
\hline $7,968750 \mathrm{MHz}$ & $10 \mathrm{MHz}$ & $-72 \mathrm{dBm} / \mathrm{Hz}$ & $84 \mathrm{Mbps}$ \\
$19,062500 \mathrm{MHz}$ & $30 \mathrm{MHz}$ & $-77 \mathrm{dBm} / \mathrm{Hz}$ & $205 \mathrm{Mbps}$ \\
\hline
\end{tabular}

\section{BROADBAND COMMUNICATION FIELD TRIAL SETUP}

The field trial environment is described in this Section. Representing part of the distribution grid of a Brazilian producing petroleum industry, it is a MV power grid composed by $1.6 \mathrm{Km}$ of four wires (three phases + a reference) with some derivations emulating a multipath fading scenario with inherits attenuation and frequency selectivity. Its tree-like topology is shown in Fig. 2.

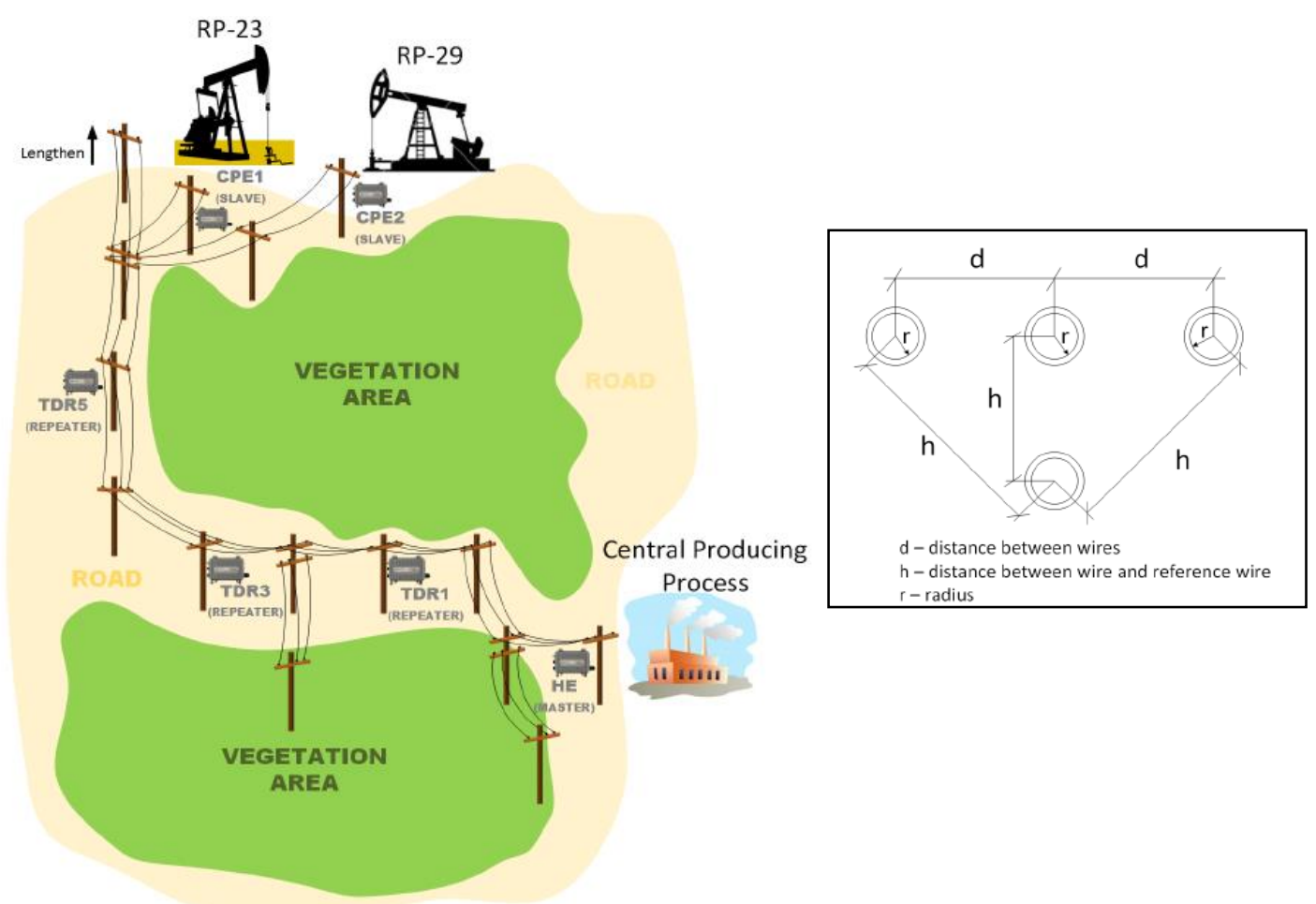

Fig. 2. The MV topology of the power distribution grid used in field trial as a BPL data transport in a smart grid concept. Inset shows the four wires layout.

The central producing process showed in Fig. 2 is the monitoring and control place of the distributed oil wells, responsible for hydrocarbon production. Therefore, the HE node that commands the BPL's network trial is located there. RP13 and RP29 represent producing units (oil wells) where the CPEs are located. The MV power distribution exhibited inset of Fig. 2 is responsible to supply medium voltage $(13.8 \mathrm{KV})$ for the pumps and, in our investigations, used as communication channel for broadband power line video and automation data transport network in a smart grid way.

It is worth to notice the main reasons that led to the choice of PLC technology in detriment of wireless and fiber based communication systems in the aforementioned case study. The foremost reason lies in the fact that we're dealing with flooded rural areas (water mirror) covered by a large volume of vegetation and with a large amount of eucalyptus trees that certainly will degrade any type of wireless communication system. Furthermore, frequently high, very and ultra high frequencies (HF, VHF and UHF) radio communication between maintenance crews also corroborates with the 
idea. The costs involved in the implementation of fiber based technologies are prohibitive because of the cost-affectivity required by the project deployment, despite the throughput required by services involved in patrimonial security.

\section{A. The BPL Network Setup}

High spectral efficiency and physical channel adaptivity through automatic gain control (AGC) algorithms, adaptive subcarrier mapping and spectrum mask are some of the majors functionalities of the acquired BPL modems [12], [13]. In order to verify their inherent control plane in physical layer for both 10 and $30 \mathrm{MHz}$ bandwidths, we conduct experimental transmission in the tree-like configuration shown in Fig. 3, in which the HE is connected to the CPEs via three TDRs.

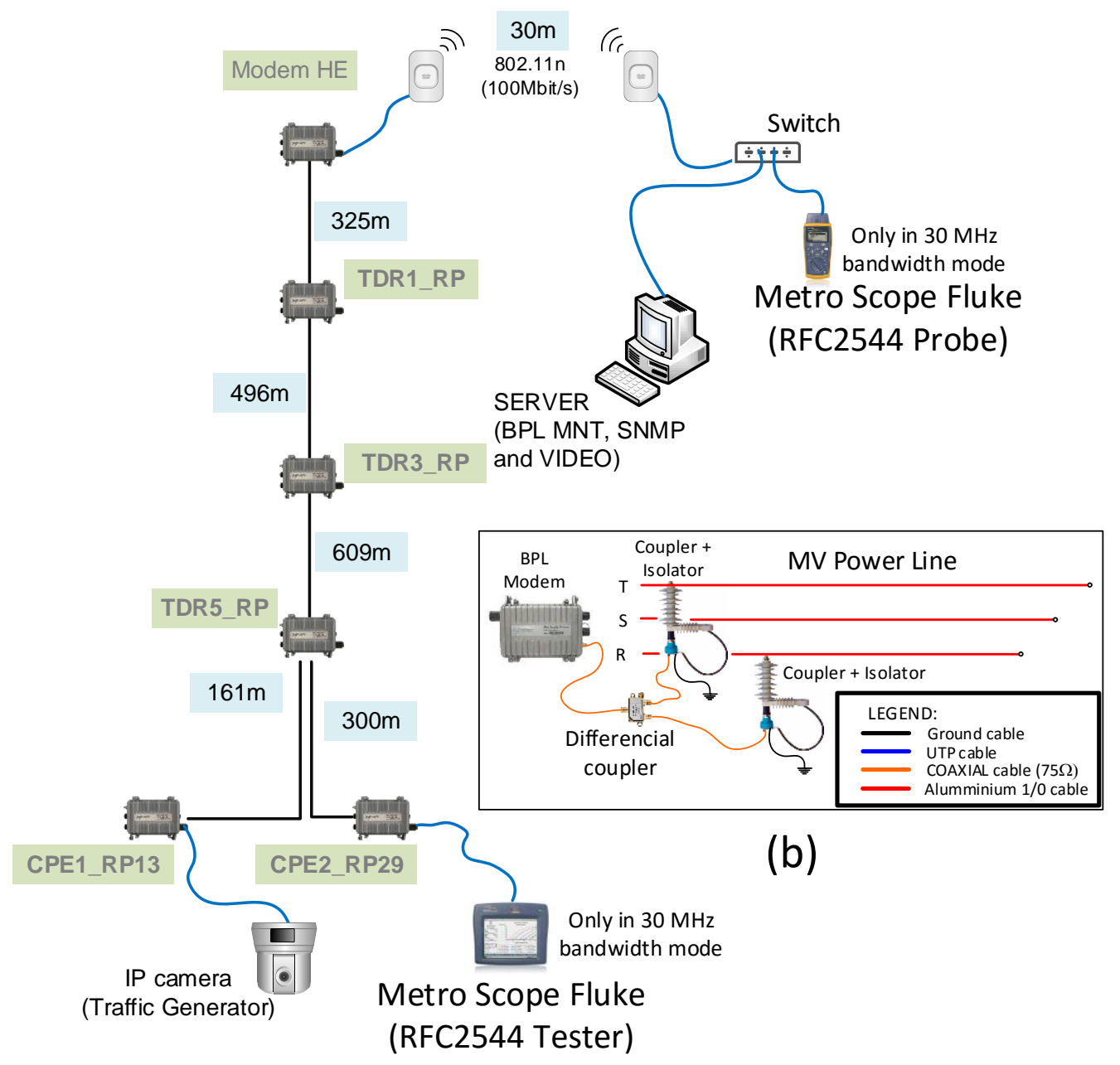

(a)

Fig. 3. a) Broadband power line network topology of the field trial. b) BPL signal coupling in MV power line.

In the first trial we investigate experimental transmission in a bandwidth of $10 \mathrm{MHz}$. As a traffic generator we used a Full High Definition Speed Dome camera plugged at the CPE located at RP13, configured to 15 frames/s, $720 \mathrm{p}$ resolution and $1536 \mathrm{~Kb} / \mathrm{s}$ in a constant bit rate mode. The camera is equipped with pantiltzoom (PTZ) functionality and it records video streams. 
The Server plugged at the HE executes three essential functions in the field trial: video server (VMS), simple network management protocol server (via a Paessler router traffic grapher PRTG application) and BPL management system known as SGNetman. The VMS is used to build a video stream from CPE to HE for throughput measurement purpose. The SNMP tool is used to get messages from BPL nodes according to its management information base (MIB) structure file, and the BPL management tool is used to manager (monitoring and control) the network, and to estimates the physical signal-to-noise ratio (SNR) of the BPL nodes. A wireless local area network (WLAN) is used to link the HE to the Server.

The PLC signal coupling in the MV power line was done by capacitive filters and $20 \mathrm{KV}$ isolators. The connection between a BPL equipment and a coupler is done by means of coaxial cables $(75 \Omega)$, as shown inset of Fig. 3.b) [13]. It should be noticed that the PLC signal was injected in two lines using a differential coupler.

In the $30 \mathrm{MHz}$ bandwidth field trial we added the RFC2544 tester which is composed by a Metro Scope Fluke tester, a reflector unit and a switch. The main goal in this investigation is to evaluate network latency and bit error rate (BER).

\section{B. The BPL Logical Topology}

The field trials logical topology is shown in Fig. 4. All devices in the BPL cell, including the BPL nodes, are connected in the same Internet protocol (IP) network. The traffic applications such as SNMP packet, BPL management packet and data/video stream packet, share the same bandwidth, with best effort profiles. In this scenario, latency sense application such as video stream or in-band management process can present bad behaviors in the case where the user traffic increases to values over the throughput limit. In our experimental investigations, the network management (BPL and SNMP management tools) works as in-band channels and, therefore, susceptible to faults.

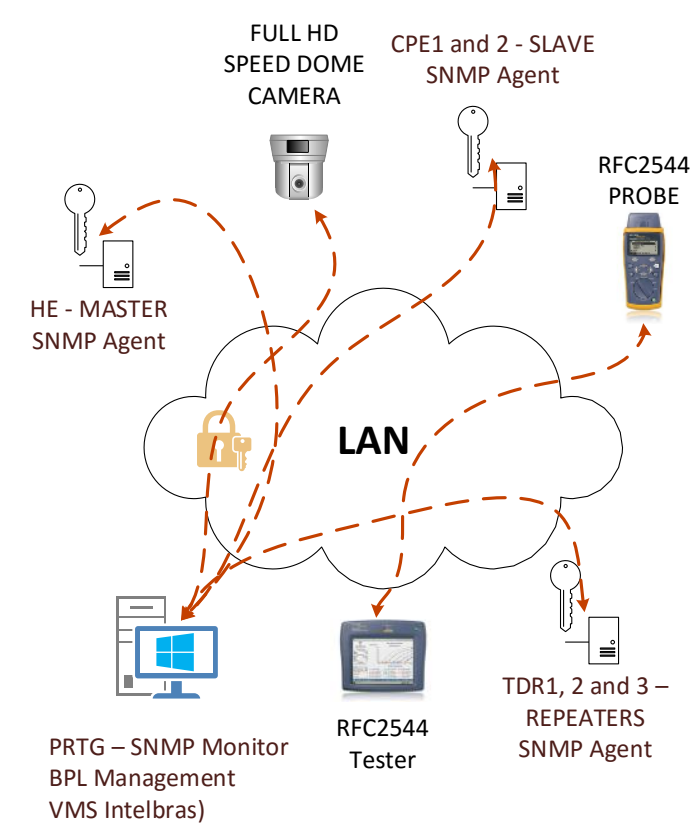

Fig. 4. The broadband power line logical topology of the field trials. 


\section{EXPERIMENTAL RESULTS}

In this section we discuss the experimental results in order to show the suitability of broadband power line technology for video and automation data transport over MV power line in the conception of a smart grid in Brazilian Onshore Oil \& Gas Industries. Throughput, latency, BER and SNR are the metrics used to evaluate the robustness of such solution.

\section{A. Physical Layer Analysis}

The measured time domain signal depicted in Fig. 5.a) shows that, even without traffic in the channel the master HE maintain a control communication with the slave equipments connected in the grid. In this case, a type of start of communications (SOT) packet is transmitted in downlink direction in order to manage the medium access of all CPEs plugged in the network. With time duration around $324 \mu \mathrm{s}$, those SOTs are spaced by $\approx 1.3 \mathrm{~ms}$ as shown in Fig. 5.b). When packets data units (PDU) are transmitted in a frame mode, a payload is observed in the physical level as depicted in Fig. 5.c).

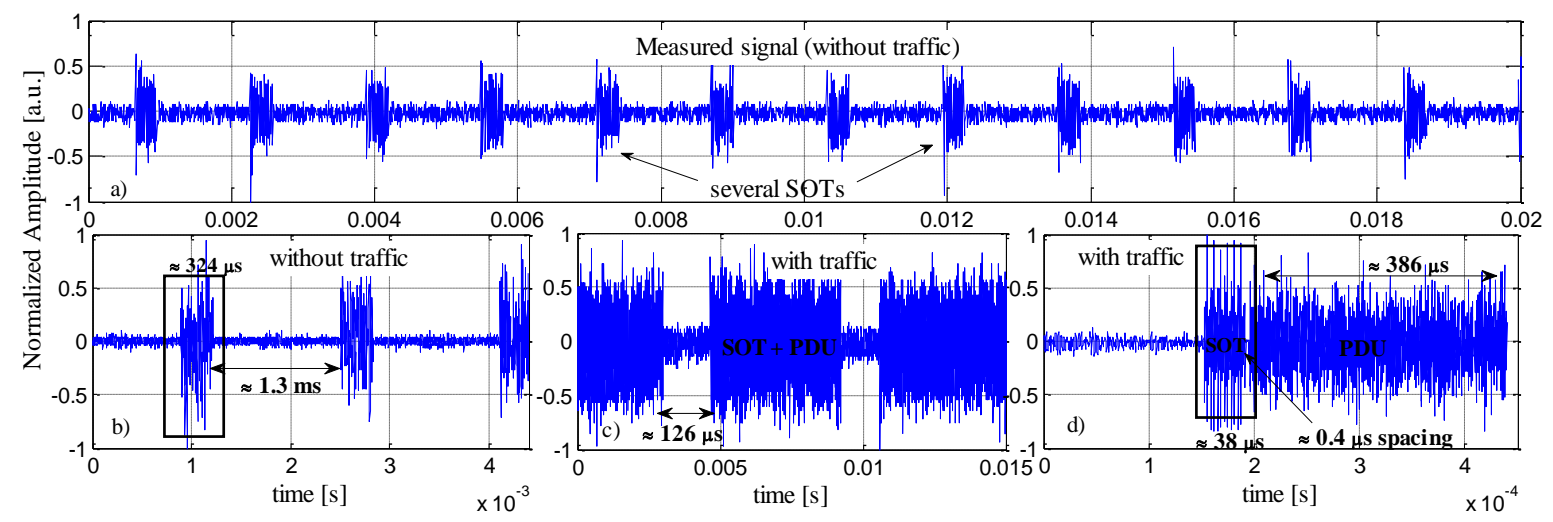

Fig. 5. Time domain signals measured at the CPE. a) and b) are without data traffic. c) and d) with traffic.

It can be seen from Fig. 5.c) that the packets composed by the SOT and payloads (synchronism, channel reference symbol and data signals) are spaced by $126 \mu$ s [11]. Separated by $0.4 \mu \mathrm{s}$ a SOT and a PDU are well established in the zoom shown in Fig. 5.d). As described in [14], the $38 \mu$ s SOT duration is composed by 6 OFDM symbols. They are useful in ACG mechanism and are transmitted before the synchronization symbol.

\section{B. Throughput Measured at Ethernet Interface}

Connected to the CPE located at RP13 (see Fig. 3), the full HD camera was used in this measurement as a traffic generator. The video streaming generated by the camera is received in the HE side by the video management Server. Fig. 6 show almost 30 days of incoming and outgoing throughput measured at the Ethernet interface of both HE and the CPE in RP13. In the HE side, the outgoing lines of Fig. 6 represent the traffic from the HE to a data terminal equipment (DTE), which is the SNMP Server in this case. The incoming lines represent traffic flow from this specific DTE to the Head end. In the CPE, the incoming represent the traffic flow from the camera to its input, while the outgoing lines represent the opposite flow. 


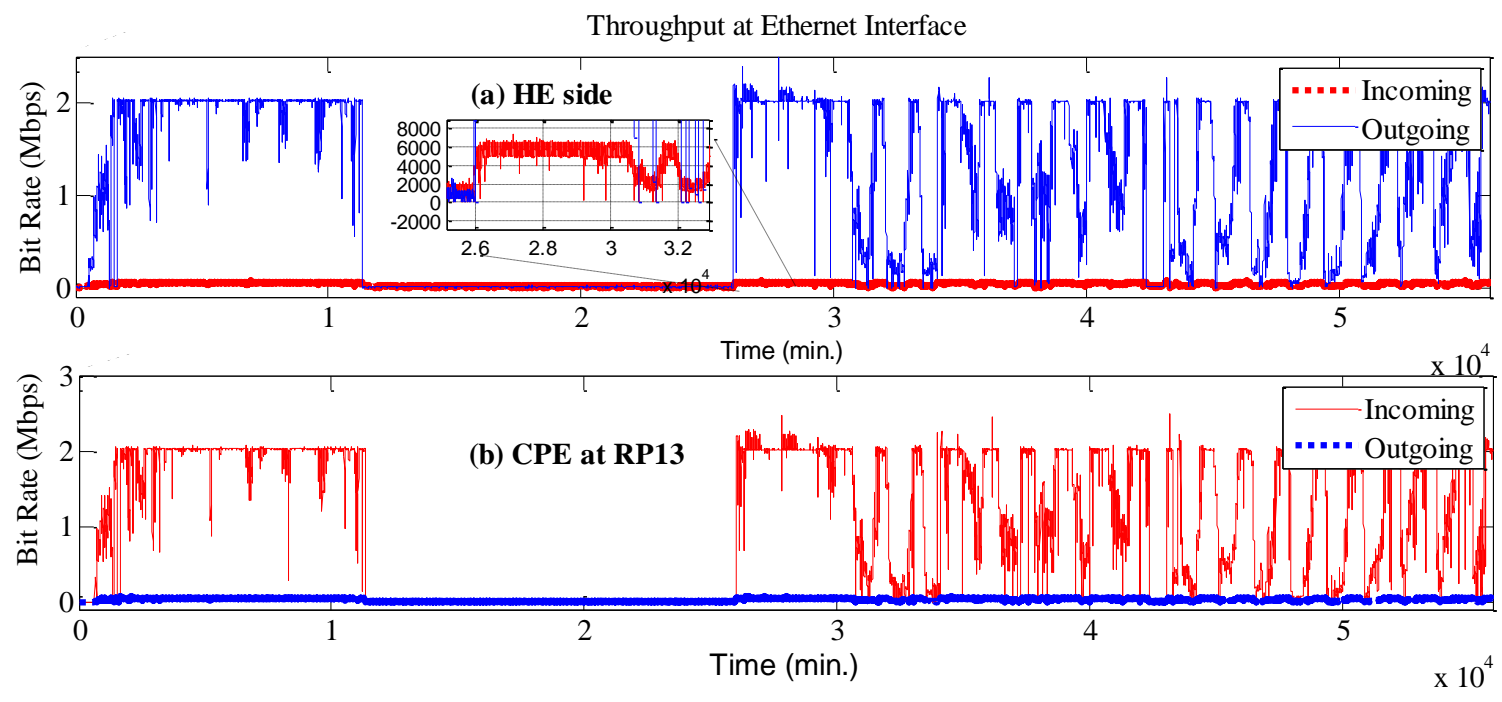

Fig. 6. Throughput measured at Ethernet interface in the $10 \mathrm{MHz}$ bandwidth mode. (a) Incoming and outgoing throughput at $\mathrm{HE}$ and (b) at the CPE in RP13.

The first ten thousand minutes shown in Fig. 6.a) reveal an average $2 \mathrm{Mb} / \mathrm{s}$ constant bit rate (CBR) traffic profile, indicating real time video streaming (including the PTZ function) without errors. As expected, the HE incoming throughput is low (see inset of Fig. 6.a)), because of the low quantity of information (frame actualization, camera control, etc) exchanged in its connection to the DTE. It should be noticed that this behavior is exactly the same in the CPE incoming, as depicted in Fig. 6.(b).

The bit rate discontinuities observed in Fig.6.a) and b) represent an energy fault and therefore, shall be ignored in the analysis. After $30^{\text {th }}$ thousand minutes of measurements, a variable bit rate (VBR) traffic profile was adjusted in the camera. The video services keep on working with a bit rate close to $2 \mathrm{Mb} / \mathrm{s}$ when the transmission is necessary. Therefore, we conclude from this result, that this technology also meets the automation application requirements.

\section{Bit Rate and Signal-to-Noise Ratio Measurements}

The success of the experimental results described in the previous Section can be explained by a performance examination of the technology in the physical layer. Hence, bit rate and signal-to-noise ratio measurements were performed at the PLC interface of the BPL equipments. Regarding to the traffic generated at the CPE in RP13, Fig. 7. a) and (b) show the system performance in downlink and uplink at the HE and the repeater TDR1 (see Fig. 3), respectively. It can be seen from Fig. 7 that for a bandwidth of $10 \mathrm{MHz}$, an average bit rate close to $19 \mathrm{Mbps}$ was registered along almost 30 hours of downlink and uplink measurements in both HE and TDR1. The bandwidth switching to $30 \mathrm{MHz}$ increases the system performance to values between 50 and $100 \mathrm{Mbps}$ in both BPL equipments. As expected, these results corroborate the experimental values measured at the Ethernet interface.

The bit rate variation observed in the $30 \mathrm{MHz}$ measurements of Fig. 7 can be explained by the adaptive bit mapping technique of the OFDM modulation implemented in the modems for channel capacity increase, according to the signal-to-noise ratio of each subcarrier. Figure 8 shows SNR measurements obtained at PLC interface of the employed BPL equipments. 

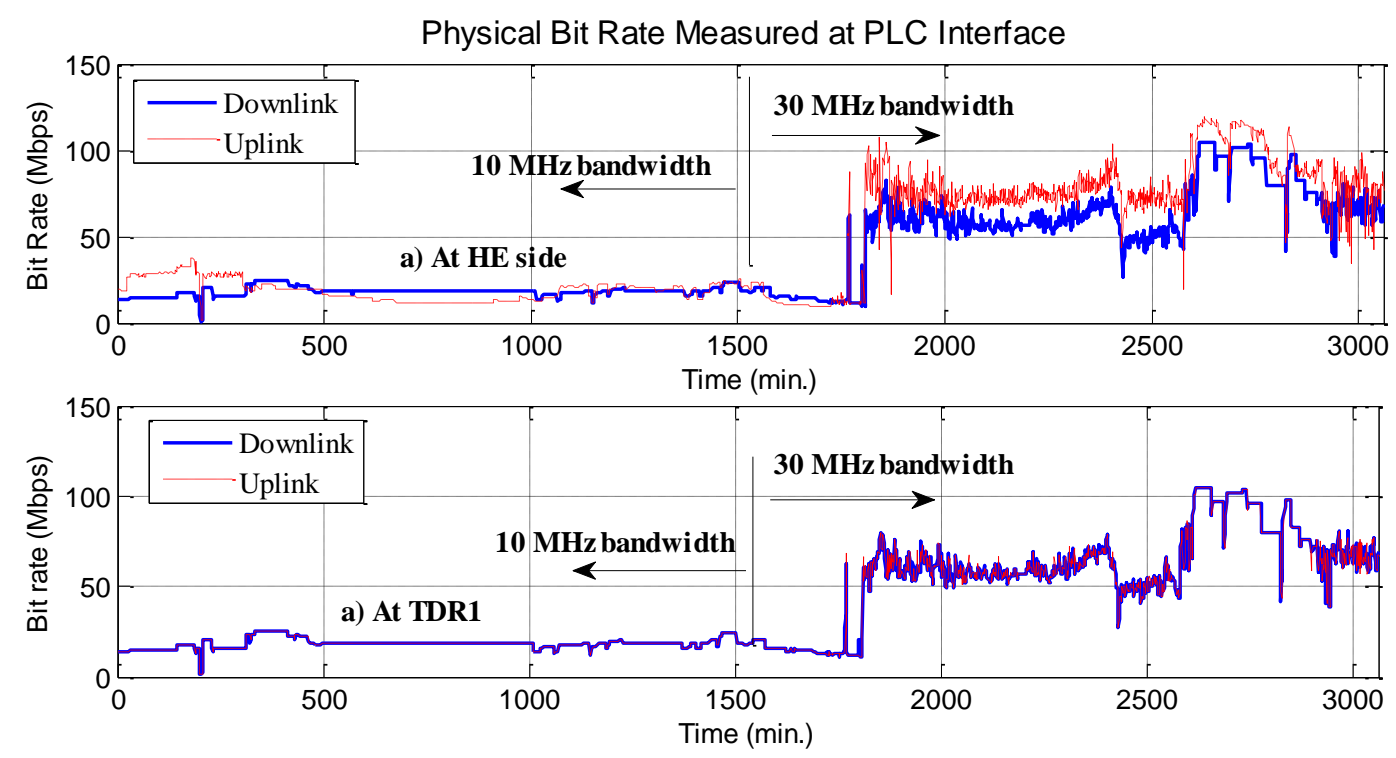

Fig. 7. System performance in terms of bit rate (in bps) at physical layer in 10 and $30 \mathrm{MHz}$ signal bandwidth, measured at PLC interface. a) At He side and b) at the time division repeater TDR1.

The time variant frequency selectivity registered in both 10 and $30 \mathrm{MHz}$ signal bandwidths of Figs. 8.a) and 8.b), respectively, reveal the necessity of an optimization in the BPL channel capacity, through a variation in the subcarriers mapping, as is in bit loading algorithms [5], [14], [15].

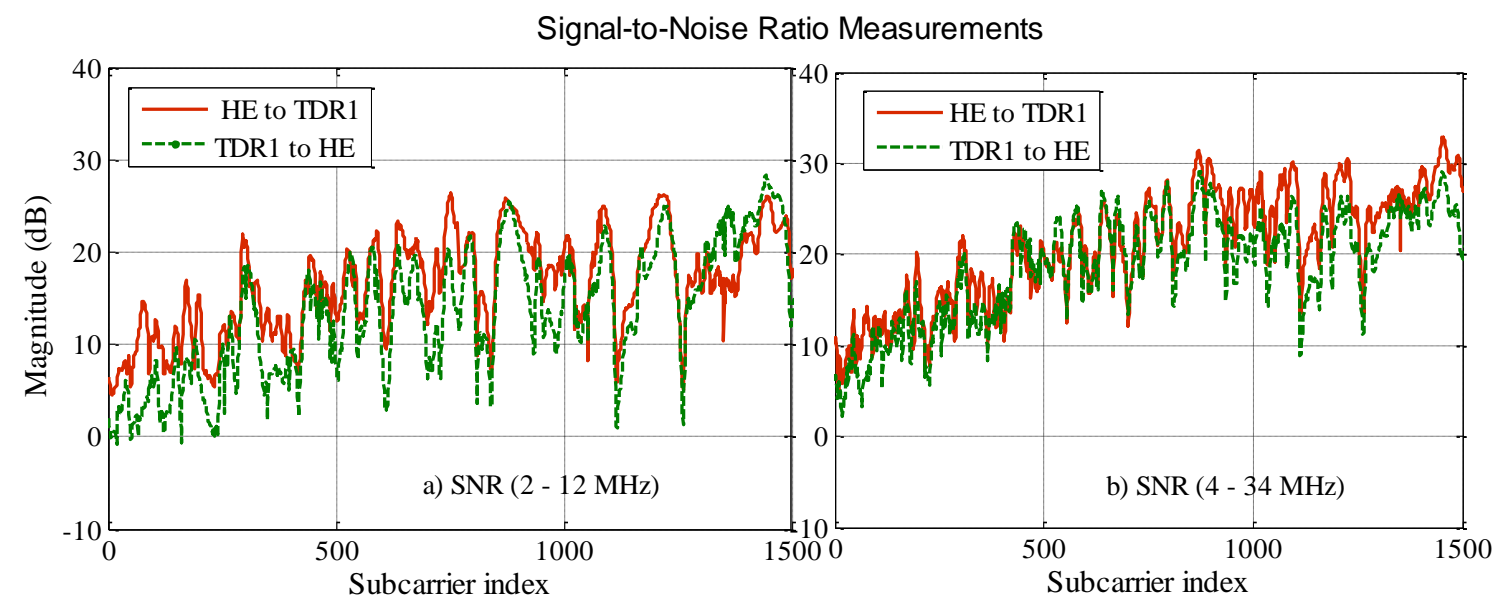

Fig. 8. SNR measurements obtained at the HE and TDR1 PLC interfaces for a) $10 \mathrm{MHz}$ and b) $30 \mathrm{MHz}$ signal bandwidths.

\section{Latency, Jitter and Bit-error-rate Measurements}

In order to analyze the latency and jitter in the BPL network, we prepared a setup using a Metro Scope Fluke instrument plus a Reflector unit as shown in Fig. 3. We noticed that those measurements are in compliance with the RFC-2544 standard. The goal is to measure the time required for frame propagation from the $\mathrm{CPE}$ to the $\mathrm{HE}$ node. This test was configured to measure the round-trip time (RTT), i.e., the time required for frame propagation from the CPE to the HE and then back to the $\mathrm{CPE}$. It should be stressed that long latencies can degrade real-time Ethernet services and client-server applications such as automation system. In a similar way, latency time variations from frame to frame cause serious issues in real-time services. 
The procedures started by benchmark testing in the throughput of each frame size to ensure that the frames were transmitted without any discard. Filling all BPL device buffers, we ensure latency measurements in the worst conditions. In a second instance, the Metro Scope Fluke instrument sends information traffic to the reflector during 120 seconds. At a transmission mid-point, a frame is tagged with a time-stamp and when it is received back at the Fluke instrument, the latency is measured. The transmission continues in the rest of the time period. Fig. 9 depicts the latency measured in the test.

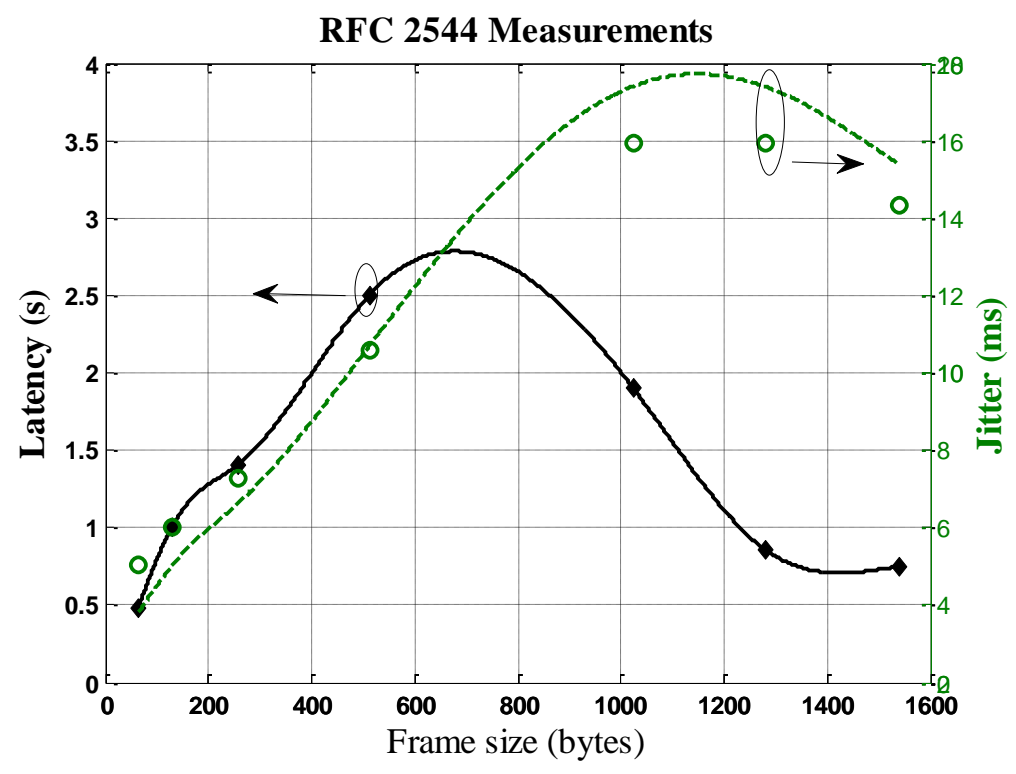

Fig. 9. Latency and jitter measurements in the BPL network provided by a Metro Scope Fluke instrument according to the RFC-2544 standard.

Latency close to $2.8 \mathrm{~s}$ is considered a high value for real-time applications. However, it was possible to transmit video signals and even controls remotely the camera (in HD configuration) functionalities in the central producing process. Therefore, we conclude that the BPL communication system is well prepared for automation data transport too. We conjuncture that the latency will decreases in future tests with the QoS functionality implemented in BPL modems.

Fig. 9 also shows the jitter as a complementary measurement. Excessive frame latency variation can cause major failures in real-time video streaming, like those observed in pixelated images. A jitter value closes to 16 ms shown in Fig. 9 do not represent impairment in the BPL communication.

\section{CONCLUSIONS}

An experimental analysis of the broadband power line communication technology employing 200 $\mathrm{Mb} / \mathrm{s}$ modems for video and automation data transport over a $1.6 \mathrm{~km}$ medium voltage distribution grid was presented in this paper. The field trial results demonstrated the potential of BPL over MV power lines as a transport medium in a smart grid communication concept for Oil \& Gas industries.

The intelligent spectrum mask and adaptive bit loading scheme, both supported by the multicarrier modulation technique, are essential characteristics to maximize channel capacities and therefore, ensures a minimal throughput for the monitoring and control of oil-wells producing processes. Indeed, 
signal-to-noise-ratio measurements denunciates an intelligent spectrum mask supported by the OFDM modulation scheme implemented in the PLC modems in order to maximize channel capacity in an adaptive way. This is useful for future Smart Grid applications in a MV network that dynamically allocates bandwidth to a broad range of interconnected secondary substations.

\section{ACKNOWLEDGMENT}

The authors acknowledge support from the PETROBRAS 2012/00186-4 project.

\section{REFERENCES}

[1] H. C. Ferreira, L. Lampe, J. Newbury and T. G. Swart, "Power Line Communications: Theory and Applications for Narrowband and Broadband Communications over Power Lines," John Wiley \& Sons, 2010.

[2] K. Dostert, "Power Line Communications," Prentice Hall, 2001.

[3] Leonardo R. M. Castor, J. A. L. Silva, M. E. V. Segatto, Ricardo Natale, "Experimental Investigation of Broadband Power Line Communication Modems for Onshore Oil \& Gas Industry: A Preliminary Analysis, " in 18th IEEE International Symposium on Power Line Communications and its Applications - ISPLC, Glasgow, 2014.

[4] M. Gotz, M. Rapp, K. Dostert, "Power line channel characteristics and their effect on communication system design," in IEEE Commun. Mag., vol. 42, no. 4, Apr. 2004, pp. 78-86.

[5] J. A. L. Silva, M. E. V. Segatto, E. O. T. Salles, T. M. Siqueira, M. N. Ribeiro, and R. V. Andreo, "A Comparative Analysis of the Performance of OFDM and COFDM Indoor PLC Systems," in 13th International Conference on Telecommunications, Funchal, 2006.

[6] X.-y. Wang, X. Gao, "The Typical Design of PLC Networks in MV Distribution Network," in IEEE International Symposium on Power Line communications and Its Applications, Beijing, 2012.

[7] X.-y. Wang, X. Gao, "The Analysis and Measurement of Transmission Characteristics and interference over MV Distribution Line," IEEE International Symposium on Power Line communications and Its Applications, Beijing, 2012.

[8] Xavier Carcelle, Power Line Communications In Practice, ISBN 13: 978-1-59693-335-4, Paris, France: Artech House, 2006.

[9] J. Anatory, N. Theethayi, Broadband Power-Line Communication Systems: Theory and Applications, ISBN: 978-184564-416-1, Boston: WIT Press, 2010.

[10] Halid Hrasnica, Abdelfatteh Haidine, Ralf Lehnert, Broadband Powerline Communications Networks: Network Design, ISBN 0-470-85741-2, Chichester, England: John Wiley and Sons Ltd, 2004.

[11] EDEV CPL/ EDF Group, "First Draft of the OPERA Specification Version 2,” EC/IST FP6 Project No $026920,2007$.

[12] Wenfei Zhua, Xu Zhub, Enggee Lima, Yi Huangb, "State-of-art Power Line Communications Channel Modelling," in Conference on Information Technology and Quantitative Management - ITQM 2013, Elsiever, 2013.

[13] P. Achaichia, M. Le Bot, P. Siohan, "Potential Impact of the CENELEC Spectral Mask on Broadband PLC Networks," in IEEE International Symposium on Pwer Line communications and Its Applications, Johannesburg, 2013.

[14] Corinex SmartGrid Connectivity, "BPL Communication for Demand Load Management and Advanced Metering Infrastructure (AMI) Backhaul," Available at www.corinex.com.

[15] T. R. Oliveira, W. A. Finamore and M. V. Ribeiro, "A Sounding Method based on OFDM Modulation for PLC Channel Measurement," IEEE Internat. Symposium on Power Line Communications and Its Applications, Johannesburg, 2013.

[16] J. A. Cortés, F. J. Canete, L. Díez and L. M. Torres, “On PLC Channel Models: an OFDM-based Comparison," in IEEE International Symposium on Power Line communications and Its Applications, Johannesburg, 2013.

[17] Leonardo R. M. Castor, J. A. L. Silva, M. E. V. Segatto, "Medium Voltage Overhead Power-line as a Smart Distribution Grid for Onshore Oil \& Gas Industries Automation and Broadband Data Transport," in IEEE Innovative Smart Grid Technologies-Latin America - ISGT-LA, Montevideo, 2015.

[18] R. P. Clayton, Analysis of Multiconductor Transmission Lines, 2a edição, Hoboken, New York: Wiley, 2007. 